\title{
Designing Simple Indoor Navigation System for UAVs
}

\author{
Mohamed Kara Mohamed, Sourav Patra and Alexander Lanzon
}

\begin{abstract}
The wide range of applications and configurations of UAVs raises the need for different types of navigation methodologies compared to the conventional INS/GPS system. For instance, the limitation in cost, size and weight of indoor UAVs makes conventional navigation system unsuitable for these vehicles. In addition, the INS/GPS navigation system is impractical for indoor applications because the GPS signal is not reliable in closed territories. This paper proposes a new, cost-effective and simple indoor navigation system using three laser beams fixed to the body of the UAV and shooting to the ground. Then, a camera and computer vision algorithm are used to capture the laser dots on the ground and determine their coordinates. The position of laser dots is used to obtain full information about the position and orientation of the UAV. The proposed navigation system can be classified as a vision based navigation system, yet, it does not depend highly on the quality of the video shots taken from the vision camera and does not require a heavy image processing algorithm. An illustrative simulation study is conducted to demonstrate the validity of the proposed navigation system.
\end{abstract}

\section{INTRODUCTION AND RELATED WORKS}

Research interest in Unmanned Aerial Vehicles (UAVs) has grown rapidly due to their wide range of applications [1]. Nevertheless, seeking full autonomy is one of the main factors behind the milestone developments in UAV systems [2], [3]. To achieve autonomy, the UAV needs a navigation system that gives information regarding the status of the vehicle and feeds this information to the controller for an appropriate action to be taken. The common navigation system used for large scale UAVs is the Inertial Navigation System coupled with the Global Positioning System (INS/GPS). However, for small UAVs or indoor applications, there is a need for alternative navigation strategies [3]. This is due to the fact that INS/GPS system is relatively expensive and needs a good access to the GPS signal which is not available in case of indoor areas.

The challenge of simple, less costy and efficient indoor navigation schemes has been undertaken by many UAV research groups [1], [3], [4]. Different techniques such as pressure sensors for altitude, magneto-resistive magnetometer, laser range finder, radar and ultra sound for position estimation and obstacles detection have been investigated [5], [3], [4]. In this regard, employing vision-based systems

The financial support of Aleppo University (Syria), the Engineering and Physical Sciences Research Council (UK) and the Royal Society (UK) is gratefully acknowledged.

The authors are with the Control Systems Centre, EEE, The University of Manchester, UK, Ph:+44 161306 8722, Fax:+44-161-306-8729, E-mail addresses:

Mohamed.Karamohamedepostgrad.manchester.ac.uk

Sourav.Patra@manchester.ac.uk

Alexander.Lanzon@manchester.ac.uk (computer vision systems) for autonomous navigation has attained considerable interest [4] due to the fact that vision based systems are lightweight, passive and produce rich information about the motion of the vehicle [5]. The vision based navigation algorithms were used initially for ground mobile robots and then imported to UAV systems [6]. To use vision based navigation systems, the path of the UAV needs to be known a priori. Images of the surrounding environment of the UAV's flying path are taken and analyzed to identify the basic features of this path before the flying mission commences. Then, the real time images taken from on-board camera(s) during the flight mission are compared with the visual memory of the vehicle to identify the known features and estimate the motion of the UAV. Different algorithms and schemes are developed to excel the feature detection and speed up the matching process [7], [8].

The computer vision systems might be used solely to estimate the motion and orientation of the vehicle by tracking the movement of the captured features in two consecutive shots from the on-board camera [6]. This method is complicated, involves heavy computational burden, affected highly by the quality of the image and the number of features to be analyzed, and works efficiently only in specific territories with good images. To reduce the complexity and computational costs, the image analysis is chosen in [9] to be performed on a ground station where the data is communicated from/to the UAV via a wireless link. This type of implementation reduces the autonomy of the UAV and puts the vehicle at risk in case of wireless communication failure. The visionbased information is integrated with other sensors such as GPS/gyroscope in [10] to refine and correct the estimation of the UAV's motion. This again requires a good GPS signal and is impractical for indoor applications or urban territories. To sum up, computer vision based systems are still developing and have many software and hardware difficulties.

A laser based navigation scheme is developed in [11] where the projection of four diode spots on the ground is used to estimate the dimensional position of the helicopter. However, the estimation of the motion of the helicopter is made by the maximum likelihood method. The algorithm contains over-determined nonlinear equations, and the authors have not discussed how to solve these equations. Moreover, the navigation system performance was not considered and rather the focus was on the control system of the helicopter.

The literature lacks such an indoor navigation strategy that is effective and easy to implement. In this work, we tackle the indoor navigation problem by introducing a new navigation strategy that benefits from the computer vision algorithms, 
but in the same time is cost-effective and easy to implement. The proposed navigation strategy uses the locations of three laser dots generated by the three laser beams fixed to the body of the vehicle to identify the status of the UAV without further requirement for optimization algorithm, fusing data estimation method like Extended Kalman Filter or additional GPS/IMU sensors. To capture the laser dots on the ground and analyze their coordinates, a computer vision technique is used, e.g., the Scale Invariant Feature Transform (SIFT) algorithm developed in [12]. The computer vision algorithm is needed only to identify the positions of the three laser dots, and therefore, the required image processing is easy and can be done on-board. This in turn helps to reduce the computational cost of the algorithm and increase the level of autonomy of the vehicle. Furthermore, the identification of the coordinates of the laser dots can be done by recognizing one landmark in the image which makes the quality of the image less important compared with other vision-based navigation strategies. The proposed system is developed to be used indoor, however, it can be used in outdoors areas where the UAV flies in low attitude above planar surfaces such as sport fields.

The rest of this paper is organized as follows: in Section II, a schematic design for the structure of the proposed system is explained. Section III discusses the mathematical formulation of the navigation scheme and the required steps to obtain the states of the UAV while Section IV is devoted to discuss the implementation process of the proposed strategy. A simulation study is presented in Section V to demonstrate the proposed navigation scheme. Some conclusions and remarks are drawn in Section VI.

\section{NAVIGATION System Description}

This paper introduces a new UAV navigation system for indoor applications. The introduced navigation system consists of three laser beams that are fixed at the center of the UAV body coordinate frame and they shoot downwards on the ground. Fig. 1 depicts the schematic diagram of the proposed navigation system. The laser beam vectors $u_{1}, u_{2}$ and $u_{3}$ form three laser spots on the ground $d_{1}, d_{2}$ and $d_{3}$. The global coordinates of the laser dots on the earth frame $X_{e} Y_{e} Z_{e}$ are determined by $\left(x_{i}, y_{i}, 0\right)$ where $i=1,2,3$ is the dot number. The ground represents the $X_{e} Y_{e}$ plane of the global coordinate system while the axis $Z_{e}$ is down toward the center of the earth. $a_{1}, a_{2}$ and $a_{3}$ are the lengths between the laser dots respectively as shown in the diagram. All measurements and values are considered in the earth frame $e$ unless specified and for simplicity of representation, the index $e$ will not be written. The angle between any two laser beams $\alpha \in\left(0, \frac{2 \pi}{3}\right)$ is identical and constant. When $\alpha \geq \frac{2 \pi}{3}$ the laser beams are either in the same plane of the UAV or shooting upwards opposite to the ground, which is inappropriate and not to be considered. The UAV body frame is denoted by the subscript $b$ and defined by the axes $X_{b} Y_{b} Z_{b}$ as shown in Fig. 1. For clarity of representation, the negative part of both axes $Z_{e}$ and $Z_{b}$ is drawn in this paper.
By knowing the coordinates of the three laser dots $\left(x_{i}, y_{i}, 0\right) i=1,2,3$, the proposed system aims to determine the position of the UAV in the Cartesian coordinates $\left(x_{v}, y_{v}, z_{v}\right)$ with respect to the earth frame and the attitude of the vehicle in form of the rotation angles between the earth frame $e$ and body frame $b$; roll $\phi_{v}$, pitch $\theta_{v}$ and yaw $\psi_{v}$.

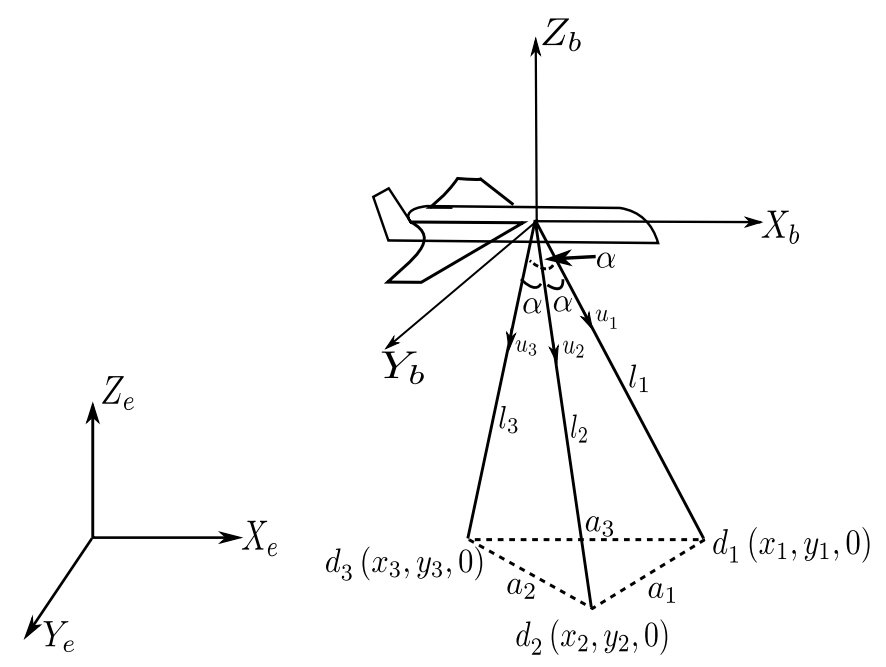

Fig. 1. The schematic diagram of the proposed navigation system.

\section{MATHEMATICAL FORMULATION}

In this work, the navigation problem is formulated as specifying the position and orientation of the UAV from the positions of the three laser dots $d_{1}, d_{2}$ and $d_{3}$. Therefore, the problem now is to obtain the vector $P=$ $\left[x_{v}, y_{v}, z_{v}, \phi_{v}, \theta_{v}, \psi_{v}\right]$ of the vehicle by knowing the positions of the three laser dots $\left(x_{1}, y_{1}, 0\right),\left(x_{2}, y_{2}, 0\right)$ and $\left(x_{3}, y_{3}, 0\right)$.

In the Cartesian space, see Fig. 1, we have:

$$
\begin{aligned}
& l_{1}^{2}=\left(x_{v}-x_{1}\right)^{2}+\left(y_{v}-y_{1}\right)^{2}+z_{v}^{2}, \\
& l_{2}^{2}=\left(x_{v}-x_{2}\right)^{2}+\left(y_{v}-y_{2}\right)^{2}+z_{v}^{2} \\
& l_{3}^{2}=\left(x_{v}-x_{3}\right)^{2}+\left(y_{v}-y_{3}\right)^{2}+z_{v}^{2} .
\end{aligned}
$$

where $l_{1}, l_{2}$ and $l_{3}$ represent the length of the laser beams. Using the cosine rule, the laser beam lengths can be calculated by solving the following nonlinear equations for the unknowns $l_{1}, l_{2}$ and $l_{3}$ :

$$
\begin{aligned}
& a_{1}^{2}=l_{1}^{2}+l_{2}^{2}-2 l_{1} l_{2} \cos \alpha, \\
& a_{2}^{2}=l_{2}^{2}+l_{3}^{2}-2 l_{3} l_{2} \cos \alpha, \\
& a_{3}^{2}=l_{1}^{2}+l_{3}^{2}-2 l_{1} l_{3} \cos \alpha,
\end{aligned}
$$

where $a_{1}, a_{2}$ and $a_{3}$ are the Cartesian distance between the laser points on the ground as shown in Fig. 1 and can be 
written as:

$$
\begin{aligned}
& a_{1}=\sqrt{\left(x_{1}-x_{2}\right)^{2}+\left(y_{1}-y_{2}\right)^{2}}, \\
& a_{2}=\sqrt{\left(x_{2}-x_{3}\right)^{2}+\left(y_{2}-y_{3}\right)^{2}}, \\
& a_{3}=\sqrt{\left(x_{1}-x_{3}\right)^{2}+\left(y_{1}-y_{3}\right)^{2}} .
\end{aligned}
$$

Solving the nonlinear Eqs. (4)-(6) to obtain the laser beam lengths is not trivial. Nonlinear equations are usually solved by using search methods. In addition to the fact that search algorithms might not converge to the correct solution, they involve computational cost [13]. In order to avoid the nonlinearity, the angle between the laser beam can be set to $\alpha=90^{\circ}$. In this case, the set of nonlinear equations is simplified and a closed form solution is obtained as:

$$
\begin{array}{r}
l_{1}=\sqrt{\frac{a_{1}^{2}-a_{2}^{2}+a_{3}^{2}}{2},} \\
l_{2}=\sqrt{\frac{a_{1}^{2}+a_{2}^{2}-a_{3}^{2}}{2}}, \\
l_{3}=\sqrt{\frac{-a_{1}^{2}+a_{2}^{2}+a_{3}^{2}}{2}} .
\end{array}
$$

Therefore, the configuration of $\alpha=90^{\circ}$ has the advantage of less computational load. However, as discussed in the next section, setting the angle between the laser beams $\alpha=90^{\circ}$ implies specific requirement for the used camera to capture the positions of the laser dots on the ground.

Solving Eqs. (1)-(3) for $x_{v}, y_{v}$ and rewriting the solution in the matrix form gives:

$$
\left[\begin{array}{l}
x_{v} \\
y_{v}
\end{array}\right]=\frac{1}{2} A^{-1} B
$$

where

$$
A=\left[\begin{array}{ll}
-x_{1}+x_{2} & -y_{1}+y_{2} \\
-x_{2}+x_{3} & -y_{2}+y_{3}
\end{array}\right]
$$

and

$$
B=\left[\begin{array}{l}
l_{1}^{2}-l_{2}^{2}+x_{2}^{2}+y_{2}^{2}-x_{1}^{2}-y_{1}^{2} \\
l_{2}^{2}-l_{3}^{2}+x_{3}^{2}+y_{3}^{2}-x_{2}^{2}-y_{2}^{2}
\end{array}\right]
$$

Subsequently, $z_{v}$ is a function of $x_{v}$ and $y_{v}$ and can be given from Eq. (1) as:

$$
z_{v}=-\sqrt{l_{1}^{2}-\left(x_{v}-x_{1}\right)^{2}-\left(y_{v}-y_{1}\right)^{2}} .
$$

The negative sign in Eq. (14) is due to the configuration of the earth coordinate system, see Fig 1. Similarly, from Eqs. (2) and (3), $z_{v}$ can be obtained as a function of the position of laser dot $d_{2}$ and the length of the laser beam vector $u_{2}$ or as a function of the position of laser dot $d_{3}$ and the length of laser beam vector $u_{3}$. Note that Eq. (13) has always a unique solution, and therefore $x_{v}$ and $y_{v}$ can be obtained always when $l_{i}, x_{i}$ and $y_{i}, i=1,2,3$ are known. The singularity in matrix $A$ occurs only when $\bar{a}_{1} \| \bar{a}_{2}$, which is not possible practically because $\bar{a}_{1}$ and $\bar{a}_{2}$ are two sides in a triangle $\left(\bar{a}_{1}\right.$ and $\bar{a}_{2}$ are the vectors between $d_{1}$ and $d_{2}$, and $d_{2}$ and $d_{3}$ respectively).

The attitude of the vehicle can be determined by obtaining the rotation matrix from the fixed earth coordinate system to the body coordinate system, see Fig. 2(a). Assuming that the

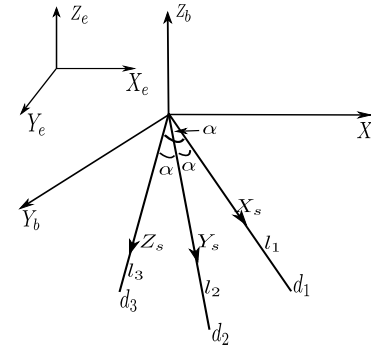

(a)

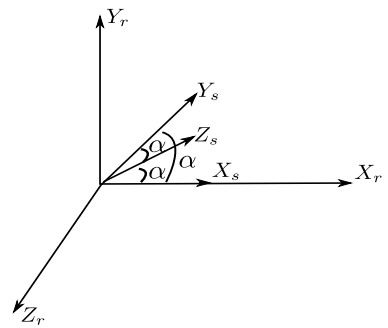

(b)
Fig. 2. The coordinate system used to develop the proposed navigation system. (a) The earth frame, body coordinate system and the laser beams coordinate system. (b) Transforming the laser beams to an orthogonal coordinate systems.

center of gravity of the UAV coincides with the origin of the laser beams, then the laser beam vector $u_{i}$ in the earth frame can be given as:

$$
u_{i}=R_{b}^{e} R_{r}^{b} T_{s}^{r} u_{i}^{s}, \quad i=1,2,3
$$

where

$u_{i}^{s}$ : the laser beam vector in the laser coordinate system $s$.

$T_{s}^{r}$ : the transformation matrix from the laser coordinate system $s$ to an orthogonal coordinate system $r$.

$R_{r}^{b}$ : the rotation matrix from the orthogonal coordinate system $r$ to the body frame $b$.

$R_{b}^{e}$ : the rotation matrix from the body frame $b$ to the earth frame $e$.

$u_{i}$ : the laser beam vector in the earth frame $e$. Fig. 2(a) and 2(b) show the coordinate systems used to develop Eq. (15).

In the Cartesian space, we can write:

$$
u_{1}=\left[\begin{array}{c}
x_{1}-x_{v} \\
y_{1}-y_{v} \\
-z_{v}
\end{array}\right], \quad u_{2}=\left[\begin{array}{c}
x_{2}-x_{v} \\
y_{2}-y_{v} \\
-z_{v}
\end{array}\right], \quad u_{3}=\left[\begin{array}{c}
x_{3}-x_{v} \\
y_{3}-y_{v} \\
-z_{v}
\end{array}\right]
$$

and

$$
u_{1}^{s}=\left[\begin{array}{c}
l_{1} \\
0 \\
0
\end{array}\right], \quad u_{2}^{s}=\left[\begin{array}{c}
0 \\
l_{2} \\
0
\end{array}\right], \quad u_{3}^{s}=\left[\begin{array}{l}
0 \\
0 \\
l_{3}
\end{array}\right]
$$

where $l_{1}, l_{2}$ and $l_{3}$ are the lengths of laser beams respectively.

From Eq. (15), we write:

$$
U=R_{b}^{e} R_{r}^{b} T_{s}^{r} U^{s}
$$

and equivalently this leads to:

$$
\begin{aligned}
R_{b}^{e} & =U\left(R_{r}^{b} T_{s}^{r} U^{s}\right)^{-1} \\
& =U\left(U^{s}\right)^{-1}\left(T_{s}^{r}\right)^{-1}\left(R_{r}^{b}\right)^{-1}
\end{aligned}
$$

where $U=\left[\begin{array}{lll}u_{1} & u_{2} & u_{3}\end{array}\right]$ is a matrix of the laser beam vectors in the earth frame and $U^{s}=\left[\begin{array}{lll}u_{1}^{s} & u_{2}^{s} & u_{3}^{s}\end{array}\right]$ is a matrix of the laser beam vectors in the laser frame $s$. Practically speaking, $U$ and $U^{s}$ exist always and they are nonsingular.

Without loss of generality, the orthogonal coordinate systems $r$ can be chosen such that $X_{r}$ is aligned with $X_{s}$, and 
$Y_{r}$ is in the plane $X_{s} Y_{s}$ as shown in Fig. 2(b). Then, in order to transfer any vector $\left[\begin{array}{lll}x_{s} & y_{s} & z_{s}\end{array}\right]^{T}$ from the laser beams coordinate $s$ to a new vector $\left[\begin{array}{lll}x_{r} & y_{r} & z_{r}\end{array}\right]^{T}$ in the orthogonal coordinate systems $r$, we write:

$$
\left[\begin{array}{l}
x_{r} \\
y_{r} \\
z_{r}
\end{array}\right]=\left[\begin{array}{lll}
t_{11} & t_{12} & t_{13} \\
t_{21} & t_{22} & t_{23} \\
t_{31} & t_{32} & t_{33}
\end{array}\right]\left[\begin{array}{l}
x_{s} \\
y_{s} \\
z_{s}
\end{array}\right]
$$

where $t_{i j}, i, j=1,2,3$ represent the elements of transformation matrix $T_{s}^{r}$ from frame $s$ to frame $r$. With the choice of the coordinate frame $r$ as in Fig. 2(b), the transfer matrix $T_{s}^{r}$ is given as (see the Appendix for details of the derivation):

$$
T_{s}^{r}=\left[\begin{array}{ccc}
1 & \cos \alpha & \cos \alpha \\
0 & \sin \alpha & \frac{\cos \alpha(1-\cos \alpha)}{\sin \alpha} \\
0 & 0 & \frac{\sqrt{1-3 \cos ^{2} \alpha+2 \cos ^{3} \alpha}}{\sin \alpha}
\end{array}\right], \alpha \in\left(0, \frac{2 \pi}{3}\right)
$$

Eq. (22) shows clearly that $T_{s}^{r}$ is not related to the status of the vehicle and therefore it is always fixed.

$R_{r}^{b}$ represents the rotation matrix from the orthogonal system $r$ to the body frame $b$. This rotation matrix is related only to the physical angles between the laser beams and the body of the UAV and therefore it is constant. To obtain $R_{r}^{b}$, we assume there is no rotation between the global earth system and the zero-time body frame, i.e., the vehicle is in a horizontal alignment with the ground with no yaw rotation before any change in the attitude of the vehicle. At this point of time, $R_{b}^{e}$ is a unity matrix, i.e., $R_{b_{0}}^{e}=I_{3 \times 3}$. Now, from Eq. (18) we can write:

$$
R_{r}^{b}=R_{r}^{b_{0}}=U_{0}\left(U_{0}^{s}\right)^{-1}\left(T_{s}^{r}\right)^{-1}
$$

where $U_{0}$ and $U_{0}^{s}$ represent the matrices of the laser beam vectors in the earth frame and laser frame respectively before the vehicle makes any move.

Now, substituting Eq. (23) in Eq. (20) gives:

$$
\begin{aligned}
R_{b}^{e} & =U\left(U^{s}\right)^{-1}\left(T_{s}^{r}\right)^{-1}\left(R_{r}^{b}\right)^{-1} \\
& =U\left(U^{s}\right)^{-1}\left(T_{s}^{r}\right)^{-1}\left(U_{0}\left(U_{0}^{s}\right)^{-1}\left(T_{s}^{r}\right)^{-1}\right)^{-1} \\
& =U\left(U^{s}\right)^{-1}\left(T_{s}^{r}\right)^{-1} T_{s}^{r} U_{0}^{s}\left(U_{0}\right)^{-1} \\
& =U\left(U^{s}\right)^{-1} U_{0}^{s}\left(U_{0}\right)^{-1}
\end{aligned}
$$

The standard orientation angles are defined as the rotation angles from $e$ to $b$ and this means that the required rotation matrix is $R_{e}^{b}$. From Eq. (27), we have:

$$
R_{e}^{b}=\left(R_{b}^{e}\right)^{-1}=U_{0}\left(U_{0}^{s}\right)^{-1} U^{s} U^{-1}
$$

The general form of the rotation matrix using the rotation angles $\phi, \theta$ and $\psi$ is given in [14] as:

$$
R=\left[\begin{array}{ccc}
C_{\theta} C_{\psi} & C_{\theta} S_{\psi} & -S_{\theta} \\
-C_{\phi} S_{\psi}+S_{\phi} S_{\theta} C_{\psi} & C_{\phi} C_{\psi}+S_{\phi} S_{\theta} S_{\psi} & S_{\phi} C_{\theta} \\
S_{\phi} S_{\psi}+C_{\phi} S_{\theta} C_{\psi} & -S_{\phi} C_{\psi}+C_{\phi} S_{\theta} S_{\psi} & C_{\phi} C_{\theta}
\end{array}\right]
$$

where $C_{x}=\cos x, S_{x}=\sin x$ and the sequence of the rotation is considered around $Z$ axis with angle $\psi$ and then around the $Y$ axis with angle $\theta$ and finally around the $X$ axis with angle $\phi$; i.e., $R=R(X, \phi) R(Y, \theta) R(Z, \psi)$. Matching the rotation matrix $R_{b}^{e}$ in Eq. (28) with the general form of the rotation matrix in Eq (29) gives the required attitude angles $\phi, \theta$ and $\psi$.

\section{IMPLEMENTATION}

The previous section shows that the position and orientation of the UAV can be determined when the information about the global position of the three laser dots is available. In order to identify the global coordinates of the laser dots, different techniques can be used. For instance, a fixed camera at the flying area can be used to capture the image of the UAV's flying path along with the laser dots, analyze this image, determine the laser dots coordinates and then supply the required information to the UAV. This technique needs a communication between the UAV and the ground station to communicate the data of the laser coordinates, which in turn reduces the level of autonomy of the UAV. Moreover, this option can be used in small area where the whole flying path can be captured by one camera. For wide spaces, more than one camera should be used to cover the whole flying path of the vehicle and be able to determine the laser dots.

Another alternative method for obtaining the coordinates of the laser dots is to use on-board camera and do the required analysis on-board. This choice increases the independence of the vehicle and makes the system flexible to be used in different environments. In this case, the global coordinates of the laser dots are calculated by using the coordinates of predefined non movable landmarks. The global positions of landmarks should be known a priori so that they can be used as database to obtain the global position of the laser dots. In details, the process starts by capturing the three laser dots and any landmarks appearing in the view to the on-board camera. Then, a computer vision algorithm can be used to analyze the captured photo and identify its components. When a landmark is recognised in the photo, a mapping matrix between the local position of the landmark in the shot and its predefined global position is calculated. Given that the positions of the laser dots in the captured image relative to the image of the landmark in the same photo shot can be determined easily, the previous mapping data of the landmark positions is used to transfer the laser dots to the global frame and obtain their global coordinates. However, in order for the laser dots to be seen by the camera at all times, a restriction to the angle $\alpha$ between the laser beams applies as shown in the following subsection.

\section{Restrictions to the angle between the laser beams $\alpha$ when} using on-board camera

When using on-board camera with certain specifications, the angle $\alpha$ between the laser beams should be designed so that the camera can capture all the three laser dots on the ground at all times. In other words, the laser dots have to be inside the Field of View (FoV) of the camera. The minimum FoV occurs in the case of horizontal alignment between the camera and the captured body. For a rectilinear image camera, the FoV of any camera with a lens of focal 
length $f$ and an optical sensor dimension $s$ is illustrated in Fig. 3.

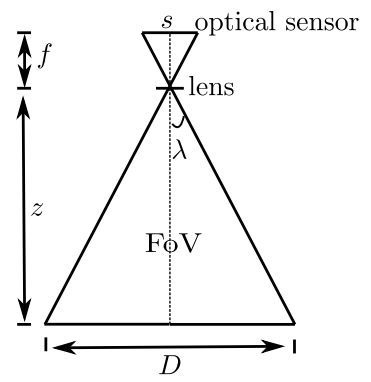

Fig. 3. The FoV of the camera.

From Fig. 3, we have:

$$
\begin{aligned}
& \tan \lambda=\frac{s / 2}{f}=\frac{D / 2}{z} \\
& \Rightarrow D=\frac{s z}{f}
\end{aligned}
$$

where $z$ is the height of the lens above $D$. For the proposed system in this paper, it is assumed that the lens of the camera is fixed at the center of the UAV and hence $z=z_{v}$. The three laser dots form a triangle of sides $\bar{a}_{1}, \bar{a}_{2}$ and $\bar{a}_{3}$ as shown in Fig. 1. This triangle must fit inside the FoV, and therefore, the circumcircle of radius $k$ that passes through the vertices of the triangle should be inside the FoV, i.e., $k \leq \frac{D}{2}$. The relation between the radius of the circumcircle $k$ and the length of the triangle sides $a_{1}, a_{2}$ and $a_{3}$ can be given [15] as:

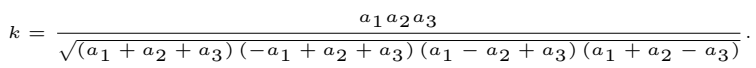

Without loss of generality, the laser system can be constructured in such a way to give $a_{1}=a_{2}=a_{3}=a$ and $l_{1}=l_{2}=l_{3}=l$ when the UAV is in horizontal alignment with the ground. In this case, we can write:

$$
k=\frac{a}{\sqrt{3}}
$$

We have $k_{\max }=D / 2$, and this leads to

$$
\begin{aligned}
D / 2 & =\frac{a_{\max }}{\sqrt{3}} \\
\Rightarrow a_{\max } & =\frac{\sqrt{3}}{2} D=\frac{\sqrt{3}}{2} \frac{s z_{v}}{f}
\end{aligned}
$$

In the horizontal alignment, the relation between $z_{v}$ and the triangle $d_{1} d_{2} d_{3}$ can be defined from Fig. 1 as:

$$
z_{v}^{2}+\frac{a^{2}}{3}=l^{2}
$$

and from the triangular relations, we have:

$$
a^{2}=2 l^{2}-2 l^{2} \cos \alpha
$$

From Eq. (36) and Eq. (37), we can write:

$$
\cos \alpha=\frac{2 l^{2}-a^{2}}{2 l^{2}}=\frac{2 z_{v}^{2}-\frac{a^{2}}{3}}{2 z_{v}^{2}+\frac{2 a^{2}}{3}}
$$

Finally, $a$ should satisfy $a \leq a_{\max }$, and this leads to:

$$
\cos \alpha \geq \frac{2 z_{v}^{2}-\frac{a_{\max }^{2}}{3}}{2 z_{v}^{2}+\frac{2 a_{\max }^{2}}{3}}
$$

Therefore, using an on-board camera of focal length $f$ and sensor dimension $s$ implies that the designed angle between the laser beams has the constraint:

$$
\cos \alpha \geq \frac{2-\frac{s^{2}}{4 f^{2}}}{2+\frac{s^{2}}{2 f^{2}}} .
$$

In the previous section, it has been shown that making $\alpha=$ $90^{\circ}$ has the advantage of simple closed solutions for the laser beam lengths $l_{i}$ and therefore it is always preferable to choose $\alpha=90^{\circ}$. In this case, the camera needs to be specifying according to this predefined angle. Using Fig. 3 with similar analysis to the above derivation leads to:

$$
\frac{s}{f} \geq 2 \sqrt{2}\left(\frac{1-\cos \alpha}{1+2 \cos \alpha}\right)^{0.5},
$$

and for the special case of $\alpha=90^{\circ}$, the chosen camera should satisfy $s \geq 2 \sqrt{2} f$.

\section{Simulation Study}

In order to demonstrate the validity of the proposed system, a brief simulation study is conducted. The position and orientation of a simulated UAV is obtained by using the laser navigation system introduced in this paper. The vehicle is flying in a fixed line along $X_{e}$ for $500 \mathrm{~s}$ at a fixed height, fixed speed and horizontal alignment with the ground without rotation, i.e., $\phi_{v}=\theta_{v}=\psi_{v}=0$. The angle between the laser beams is set to $\alpha=90^{\circ}$ and hence the length of the laser beams are obtained by using Eqs. (10)(12). Fig. 4 records the position and orientation of the vehicle during the flight time. At the time $t=100 \mathrm{~s}$, disturbances occur that push the vehicle away from its planned path along $Y_{e}$ by $1.5 \mathrm{~m}$ and the proposed navigation system records this change as shown in the plot titled "Y position". Another disturbance is imposed to change the speed of the vehicle along the $X_{e}$ in the period $t=250-350 \mathrm{~s}$ and the proposed navigation system tracks the change as shown in the plot titled "X position". In the graph " $Z$ position", the system records the height of the UAV and the change happens at $t=350 \mathrm{~s}$ from $-0.82 \mathrm{~m}$ to $-1.63 \mathrm{~m}$. Around the time $t=450 \mathrm{~s}$, disturbances are imposed to change the attitude of the vehicle for some seconds before it returns to its planned trajectory. The proposed navigation system records this change as shown in the plots titled "Roll angle", "Pitch angle" and "Yaw angle". 

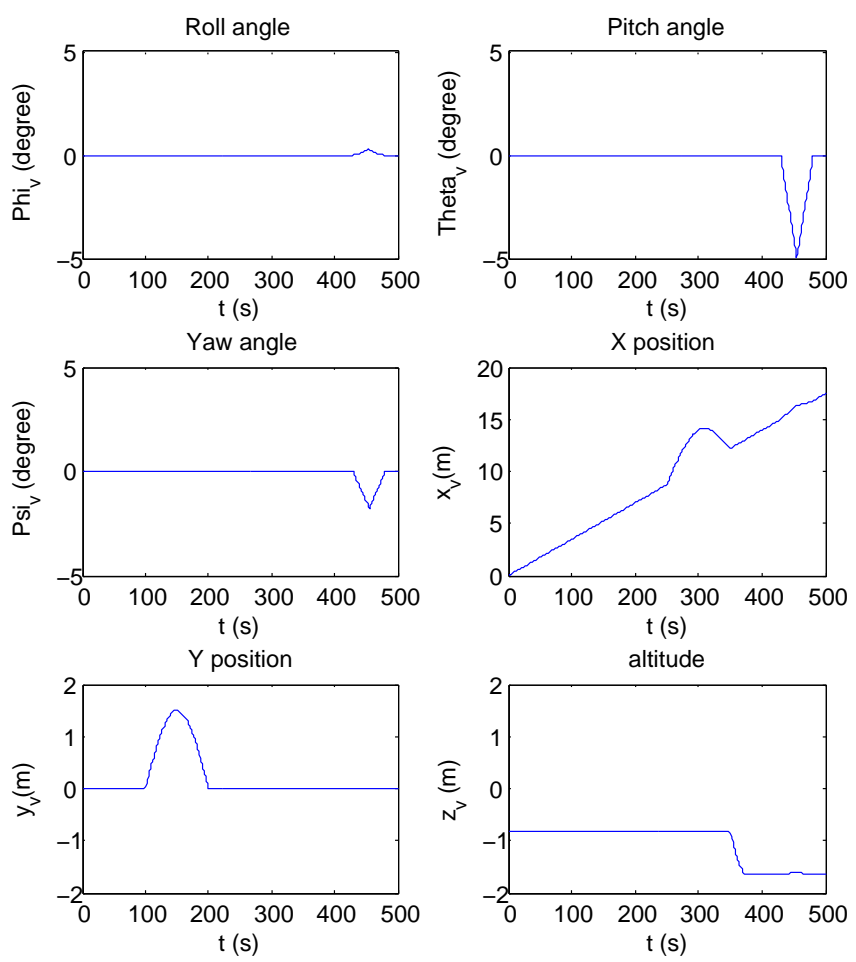

Fig. 4. Simulation study for the proposed navigation system.

\section{CONCLUSION}

This paper proposes a new, cost-effective and simple navigation system for UAVs. The system is proposed for indoor applications, yet, it can be used also in outdoor missions when the UAV flies at low altitude in plain and predetermined areas. This makes the system ideal for testing stages of mini UAV systems where the possibility of crash is high and it is preferable not to use an expensive navigation system during these flight tests. The system provides full information about the position and orientation of the UAV using three laser beams fixed to the vehicle's body system and pointing downward to the ground. A computer vision algorithm is needed to identify the dots and determine their positions. The system is more efficient when the angle between the laser beams is $\alpha=90^{\circ}$ as it becomes possible to get the length of the laser beams without the need for extra sensors or estimation algorithms. A simple simulation study is conducted to show the ability of the proposed navigation system to record the status of the UAV.

\section{APPENDIX}

To derive the transformation matrix $T_{s}^{r}$ in Eq. (22), we can write from Fig. 2(b):

$$
\begin{array}{ll}
t_{11}=1 & t_{12}=\cos \alpha \quad t_{13}=\cos \alpha \\
t_{21}=0 & t_{22}=\sin \alpha \\
t_{31}=0 & t_{32}=0
\end{array}
$$

The inner products of the unit vectors of $X_{s}$ and $Y_{s}$ gives:

$$
\left(t_{12} \cdot t_{13}\right)+\left(t_{22} \cdot t_{23}\right)+\left(t_{32} \cdot t_{33}\right)=|1||1| \cos \alpha
$$

$$
\Rightarrow t_{23}=\frac{\cos \alpha(1-\cos \alpha)}{\sin \alpha}
$$

In addition, the cosine directions of any vector is equal to 1 . This gives:

$$
\begin{gathered}
t_{13}^{2}+t_{23}^{2}+t_{33}^{2}=1 \\
\Rightarrow t_{33}=\frac{\sqrt{1-3 \cos \alpha^{2}+2 \cos ^{3} \alpha}}{\sin \alpha},
\end{gathered}
$$

and this makes the matrix $T_{s}^{r}$ as:

$$
T_{s}^{r}=\left[\begin{array}{ccc}
1 & \cos \alpha & \cos \alpha \\
0 & \sin \alpha & \frac{\cos \alpha(1-\cos \alpha)}{\sin \alpha} \\
0 & 0 & \frac{\sqrt{1-3 \cos ^{2} \alpha+2 \cos ^{3} \alpha}}{\sin \alpha}
\end{array}\right], \alpha \in\left(0, \frac{2 \pi}{3}\right)
$$

[1] K. P. Valavanis, Ed., Advances in Unmanned Aerial Vehicles State of the Art and the Road to Autonomy, ser. International Series on Intelligent Systems, Control, and Automation: Science and Engineering. Springer, 2007, vol. 33.

[2] P. Vanblyenburgh, "UAVs: An overview," Air \& Space Europe, vol. 1, no. 5-6, pp. 43-47, September 1999.

[3] S. Ehsan and K. D. McDonald-Maier, "On-board vision processing for small UAVs: Time to rethink strategy," in Adaptive Hardware and Systems, NASA/ESA Conference on, 2009, pp. 75-81.

[4] J. Zhang, Y. Wu, and W. Liu, "Novel approach to position and orientation estimation in vision-based UAV navigation," IEEE Transactions on Aerospace and Electronic Systems, vol. 46, no. 2, pp. 687-700, 2010.

[5] F. kendoul, I. Fantoni, and K. Nonami, "Optic flow-based vision system for autonomous 3d localization and control of small aerial vehicles," Robotics and Autonomous Systems, pp. 591-602, 2009.

[6] M. R. Parks, "Vision based self motion estimation in a fixed wing aerial vehicle," Master's thesis, Faculty of Virginia Polytechnic Institute and State University, 2006.

[7] J. Courbon, Y. Mezouar, L. Eck, and P. Martinet, "Efficient hierarchical localization method in an omnidirectional images memory," in IEEE International Conference on Robotics and Automation, 2008, pp. 1318.

[8] H. Bay, A. Ess, T. Tuytelaars, and L. V. Gool, "Surf: Speeded up robust features," Computer Vision and Image Understanding (CVIU), no. 3, 2008

[9] J. Courbon, Y. Mezouar, N. Nicolas Guénard, and P. Martinet, "Visionbased navigation of unmanned aerial vehicle," Control Engineering Practice, vol. 18, pp. 789-799, 2010.

[10] B. Sinopoli, M. Micheli, G. Donatoy, and T. J. Koo, "Vision based navigation for an unmanned aerial vehicle," in Proc. of the IEEE International Conference on Robotics an Automation (ICRA 2001, vol. 2, 2001, pp. $1757-1764$.

[11] A. E. Barabanov and D. V. Romaev, "Design of helicopter autopilot," in Proceedings of the European Control Conference, 2009, pp. 38393844.

[12] D. G. Lowe, "Distinctive image features from scale-invariant keypoints," International Journal of Computer Vision, vol. 60, no. 2, pp. 91-110, 2004.

[13] W. C. Rheinboldt, Methods for Solving Systems of Nonlinear Equations, $2^{\text {nd }}$ ed., ser. CBMS-NSF Regional Conference Series in Applied Mathematics 70 . Society for Industrial and Applied Mathematics(Cambridge University Press), 1998.

[14] H. Schaub and J. L. Junkins, Analytical Mechanics of Space Systems, ser. AIAA Education Series. Reston, VA, USA: AIAA, 2003, vol. 1.

[15] D. Pedoe, Geometry: A comprehensive course. Dover Publications, 1970. 